\title{
in medias res
}

\section{Zur Bedeutung von Universitätssammlungen}

Anke te Heesen

Über das fruchtbare Verhältnis zwischen Wissenschaft und Museum oder Wissenschaft und Ausstellungswesen ist in den letzten Jahren viel nachgedacht worden. Eine lebendige, seit den 1980er Jahren expandierende Sammlungsgeschichte hat Zusammenhänge deutlich werden lassen, die über eine reine Provenienzforschung oder Inventarisierungsbeschreibungen weit hinausgehen. Die neuere Wissenschaftsgeschichte hat sich intensiv mit der Wechselbeziehung von Objekten und Forschungsprozessen beschäftigt, doch ihre ertragreichen Zusammenhänge werden nur selten mit den finanziellen und organisatorischen Niederungen des universitären Sammlungserhaltes in Verbindung gebracht. Nicht nur die finanziellen Mittel sind vielerorts mehr als knapp bemessen. Es fehlt auch an Kustoden, die eine genuine Fachkenntnis der Objektkonvolute besitzen. Die schlichte Zurverfügungstellung von Räumlichkeiten, in denen die Objekte zumindest aufbewahrt werden können, grenzt oft an die Unmöglichkeit und scheitert häufig an dem notwendigen und in den Feuilletons immer wieder eingeklagten historischen Bewusstsein vor Ort. Dabei erweist es sich nicht gerade als hilfreich, wenn kulturpessimistische Sachverwalter sich als Stimmen solcher Sammlungen zu Wort melden und schimpfend für den Erhalt der historischen Objekte einstehen. Vielmehr sollte der Schwung der neueren historischen Wissenschaftsforschung mit dieser Problematik in Verbindung gebracht werden. In den größeren Wissenschaftsmuseen, wie dem Science Museum in London, dem Deutschen Museum in München oder dem Deutschen Hygiene-Museum in Dresden geschieht dies zum Teil bereits (vgl. aktuelle Ausstellungsthemen wie etwa „Atombilder. Strategien der Sichtbarmachung im 20. Jahrhundert“, 8.5.2007-27.1.2008 am Deutschen Museum). Diese Verbindung fehlt jedoch noch in vielen Fällen - und darüber mag man sich verwundert die Augen reiben - vor allem in den Universitäten selbst und in den in diesen Institutionen ruhenden sogenannten Universitätssammlungen. Es soll in diesem Beitrag darum gehen, wie einerseits die Objekte der Universitätssammlungen vor dem Hintergrund des neueren Ausstellungswesens ver- 
mehrte Beachtung erfuhren, wie aber andererseits diese Sammlungsspezies in der alltäglichen Organisation durch schärfste finanzielle Beschränkungen und ein schwindendes Institutionengedächtnis akut bedroht ist.

Unter einer Universitätssammlung versteht man im engeren Sinne eine Sammlung oder permanente Ausstellung der Insignien einer Hochschule, also die Universitätszepter, Rektorkette, Pokale, Talare, Doktorhüte, Matrikelbücher und andere wertvolle, institutionengebundene Objekte. Im weiteren und heute üblicheren Sinne wird damit neben den oben genannten Objekten vor allem der forschungs- und lehrrelevante Objektfundus der Geistes- wie Naturwissenschaften einer Universität bezeichnet, also das, was in früheren Zeiten zumeist unter dem Begriff der „Lehrmittelsammlung“ firmierte. Universitätssammlungen entstanden, weil ihre Objekte in der Lehre benötigt wurden, wie etwa mathematische Gipsmodelle in der Zeit um 1900, oder aber, weil sie eine für die Forschung relevante Rolle spielten, wie zum Beispiel die Graphiksammlung eines kunsthistorischen Instituts, oder weil sie schlicht der Selbstdarstellung eines Faches dienten. Nicht wenige dieser Objekte vor allem aus dem naturwissenschaftlichen Bereich haben vor noch nicht allzu langer Zeit erstmals wieder das Tageslicht erblickt und rückten ins Zentrum der Konjunktur wissenschaftlicher Objekte.

Eine universitätsübergreifende Organisation erfuhren diese Sammlungen um das Jahr 2000, als erstmals miteinander vernetzte Institutionen zu ihrer Erschließung und Erfassung gegründet wurden. Im Falle des „Universeum“ handelt es sich beispielsweise um ein EU-Projekt, das „the academic heritage and universities“, „responsibility and public access“ zum Thema hatte (www.universeum.de, Stand: 7/2008). UMAC - University Museums and Collections wurde als Zweig des ICOM (International Council of Museums) im Jahr 2001 gegründet, um alle Universitätssammlungen weltweit zu erfassen (http://publicus.culture.hu-berlin.de/umac/ Stand: 7/2008). Schließlich wurde mit Gründung des Hermann von Helmholtz-Zentrums für Kulturtechnik der Humboldt Universität zu Berlin das Projekt „Wissenschaftliche Sammlungen" ins Leben gerufen. Es handelt sich dabei um eine Erfassung aller universitären Sammlungen Deutschlands in einer Datenbank sowie die in der Folgezeit unternommene Entwicklung einer Maske für die digitale Aufnahme, Speicherung und Vernetzung dieses speziellen Sammlungsgutes (www2.hu-berlin.de/hzk/sammlungen.php, Stand: 7/2008).

Ausgehend von diesen jüngeren Aktivitäten fragt sich, wie das Interesse für die universitäre Sammlungsgeschichte und ihre Objekte entstand und welche historischen Hintergründe auch heute noch bei der Diskussion um das durchaus schwierig zu nennende Sachzeugenerbe („academic heritage“) eine Rolle spielen. Denn blickt man auf die gegenwärtigen Aktivitäten der Universitäten, so stehen Jubiläumsausstellungen und mit ihnen die Objekte der Sammlungen im Vordergrund (vgl. etwa Erlangen 2007, demnächst Leipzig 2008, Berlin 2010). Außerhalb der Jubiläumszeiten wird jedoch vor 
dem Hintergrund der finanziellen Möglichkeiten schnell vergessen, dass in den Universitätssammlungen auch und vor allem die Voraussetzung für eine zukünftige und programmatische Auseinandersetzung mit der materiellen Kultur der Wissenschaften liegt.

Doch noch einmal zurück zur neueren Geschichte dieses Sammlungstyps, die nicht zuletzt das besondere und wertvolle Potenzial der Bestände verdeutlicht. Den Beginn einer Entwicklung, die die Forschungs- und Lehrartefakte vermehrt in einen museumswürdigen Status überführte, markieren seit dem Ende der 1980er Jahre neuartige große Themenausstellungen. Bei den Ausstellungen "Wunderblock" in Wien (1989, kuratiert von Jean Clair, Catherin Pichler und Wolfgang Pircher), „Der Gläserne Mensch“ in Dresden (1990, kuratiert von Rosmarie Beier und Martin Roth) oder "L'âme au corps" in Paris (1993, kuratiert von Jean Clair) handelte es sich um Konzeptionen, die natur- und kulturwissenschaftliche Ansätze vereinigten. Sie erhoben den Anspruch, beide Kulturen gleichsam am Objekt dingfest zu machen - beispielsweise indem die Couch Siegmund Freuds zugleich mit einem der ersten Röntgentische präsentiert wurde, um so die geistigen wie körperlichen Durchleuchtungsbestrebungen des Menschen um 1900 sinnfällig zu machen. Hier konnte die vielbeschworene wissenschaftliche Transdisziplinarität inhaltlich und visuell eingelöst werden. Und es zeigte sich, dass auch im Ausstellungswesen Forschungsarbeit jenseits von Provenienzbestimmung und Inventarisierung zu leisten war, ja, dass die ungewöhnlichen Dingarrangements wiederum Anlass zu neuen Projektideen gaben. Mit der Wertschätzung des musealen Objekts einerseits und seiner Öffnung auf interdisziplinäre Zusammenhänge andererseits waren diese Ausstellungen nicht mit der Aufarbeitung eines bestimmten Bestandes befasst (also der bis dahin so bezeichneten genuinen Forschungsaufgabe des Museums), sondern sie sahen das Zusammenführen verschiedenster Genres und Traditionen als ihre wichtigste Aufgabe an. Dies war der Moment, an dem die Existenz von Universitätssammlungen mehr und mehr ins Bewusstsein sowohl der Ausstellungsmacher als auch der Ausstellungsbesucher trat.

Das öffentliche Interesse an den wissenschaftlichen Sammlungen auf universitärer Ebene wurde schließlich im deutschsprachigen Raum vor allem mit der umfassenden Ausstellung "Theatrum Naturae et Artis“ in Berlin geweckt (2000, kuratiert von Horst Bredekamp, Jochen Brüning und Cornelia Weber). Die Sammlungsgeschichte hatte zu diesem Zeitpunkt ihren Höhepunkt erreicht und war durch zahlreiche innovative Ansätze und Publikationen etabliert. Vor allem das Modell der Kunst- und Wunderkammer konnte ausführlich in der Forschungsliteratur und im Feuilleton der Tageszeitungen verfolgt werden. Dieses Modell wurde in Berlin programmatisch in den Raum umgesetzt. Die Ausstellungsmacher wussten die Schätze der Humboldt-Universität zu Berlin zu heben und zu inszenieren und vermochten die disparatesten Sammlungen wie die der Anatomie und der Archäolo- 
gie mit Hilfe einer inhaltlichen wie darstellungsleitenden Idee zu verbinden. Die Sammlungen wurden als eine die Fakultäten vereinigende Klammer für die Zukunft gefeiert. Seitdem fanden zahlreiche kleinere Ausstellungen an den verschiedensten Universitäten statt, die dieses Potenzial einer unter dem Etikett „Kunst und Wissenschaft" firmierenden Vereinigung zu nutzen verstanden. Rückblickend ist festzuhalten, dass die inhaltliche und präsentationstechnische Metapher der Kunst- und Wunderkammer einen wichtigen Einstieg in die Universitätssammlungen bildete. Was aber 2000 noch so neu war, dass man Sammlungen wie Kustoden selbst in einem gewaltigen Kraftakt bewunderungswürdig unter einen Hut zu bringen verstand, entspricht heute nicht unbedingt dem aktuellen Stand dieser Sammlungsart. Die Kunst- und Wunderkammer hat für einen fruchtbaren Moment den Hintergrund für die Universitätssammlungen geliefert. Dauerhaft gearbeitet werden kann damit jedoch nicht, da diese Sammlungen in Inhalt, Nutzen und Präsentationsgeschichte so verschieden sind wie nur irgend möglich. Was ist es also dann, was sie vereint und sinnvoll unter ein Dach zu bringen vermag?

Zunächst ihre gemeinsamen Schwierigkeiten. Blickt man auf die Beweggründe, die zu einer inner- wie außeruniversitären Aufmerksamkeit für die Relikte der Lehre und Forschung führen, so wird schnell deutlich, dass dabei die Möglichkeiten der Digitalisierung, also die Medienentwicklung eine entscheidende Rolle spielt. Noch nie waren in so kurzer Zeit so viele Medienbrüche und -wechsel zu verzeichnen wie in den letzten zwanzig bis dreißig Jahren. Diese Wechsel produzierten nicht nur immer mehr Instrumente und neue Objekte, sondern lassen die Räume der Institution heute geradezu in einer Objektschwemme versinken. Auch um 1900 füllten sich die Lehr- und Forschungsschränke sprunghaft. Im letzten Drittel des 19. Jahrhunderts war die Lehrmittelproduktion enorm angestiegen, neue Visualisierungs- und Materialverfahren (etwa die Herstellung von Wachsmodellen für die Anatomie) wurden umgesetzt und in Serie produziert. Auch damals gab es mitunter Kapazitätsprobleme, doch über allem lag die Hoffnung einer Wissenschaft, die immer besser zu visualisieren und weiter zu,vermehren' sei. Heute, 2008, geht es um den zeitlichen Verfall der Forschungs- wie Lehrobjekte und damit um ihre Historisierung. Ein Beispiel: Bis vor zwei, drei Jahren gab es zahlreiche Kunsthistoriker, die das klassische Dia und seine Projektion der Powerpoint-Präsentation vorzogen. Nachvollziehbar begründet wurde diese Auffassung damit, dass die Powerpoint-Präsentation noch nicht das Niveau des Dias erreicht habe, das immer noch das exaktere Zeigemedium sei. Diese Meinung hat sich indes grundlegend geändert: Inzwischen greifen selbst hartnäckigste Fachvertreter auf die digitalen Möglichkeiten zurück. Was soll nun, so fragt man sich nicht nur in zahlreichen kunsthistorischen Seminaren, mit den Tausenden Dias und ihren Leuchtkästen und Leuchtschränken geschehen, die für das Fach entscheidend waren und zugleich ganze Generationen von Lehrenden und Studierenden in ihrem Wahrnehmungsverhalten 
maßgeblich geprägt haben? Ein anderes Beispiel: Für die Pathologie spielen Objektträger aus Glas eine bedeutsame Rolle. Feinste Gewebeschnitte werden angefertigt, auf den Objektträger aufgetragen, eingefärbt und dienen so der eingehenden Untersuchung mit Hilfe des Mikroskops. Heute jedoch werden die fertigen Schnitte anschließend digitalisiert und damit zur näheren Untersuchung auf dem Computer verfügbar gemacht. Der Objektträger selbst wird, wenn überhaupt, für eine kurze Zeit archiviert und danach entsorgt. Auch hier warten Tausende alte, noch bestehende und bereits archivierte Objektträger, die bisher als notwendige materiale Referenz galten, auf eine Entscheidung über ihren weiteren Verbleib oder ihre Entsorgung.

Die angeführten Medienwechsel in Forschung und Lehre haben dafür gesorgt, dass auch die Erinnerung an das, was noch vor fünf Jahren als Lehrmittel eingesetzt wurde, mehr und mehr verblasst. Ein Historisierungsprozess der materiellen (Forschungs-)Welt hat eingesetzt, für dessen Behandlung es noch keine adäquate Lösung gibt. Alles archivieren? Nein, stattdessen differenziert auswählen - aber wie? Die Wissenschaftsgeschichte der letzten beiden Jahrzehnte hat uns gelehrt, dass kleine Notizbücher oder ein altes Reagenzglas im Nachhinein eine ungeheure Bedeutung erlangen können (nicht nur als Forschungsreliquie, sondern vor allem als aussagekräftige Quelle für eine zu rekonstruierende Forschungspraxis). Wie sieht es diesbezüglich mit dem EMail-Verkehr der Wissenschaftler und Wissenschaftlerinnen heute aus? ${ }^{1}$

Während das Museum einen Rahmen des Bewahrens, des Deponierens und Exponierens vorgibt, ist die Aufgabe der Objekte in der Universitätssammlung eine grundlegend andere: Auch hier werden Objekte archiviert und deponiert, doch sind sie in den Kreislauf der Lehre und Forschung, der beiden genuinen Aufgaben der Universität, eingebunden. Im Vordergrund stehen ihre Qualitäten als didaktisches Anschauungsmaterial und als Forschungsgrundlage. Das primäre Interesse an den Objekten ist ein aktuelles, gegenwärtiges und nicht, wie in den Museen, ein historisches. Hinzu kommt, dass es weniger um einen Sammlungskorpus geht, den man mit Hilfe von Erwerbungen zusammenzustellen oder zu ergänzen sucht, sondern vielfach um Sammlungen, die sich zum Beispiel aus dem Ankauf einer fertigen Serie (etwa Lehrmittel) oder aus dem Forschungsprozess selbst ergeben haben. Wenn in einer universitären Augenklinik eine Sammlung von "Fremdkörpern“ um die Jahrhundertwende zusammengestellt wurde, dann geschah dies nicht (nur) um der Sammlung selbst willen, sondern weil die aus den Augen herausoperierten Kleinteile als Demonstrationsstücke für die Studenten beiseite gelegt wurden. Erst mit dem zeitlichen Abstand von nunmehr fast hundert Jahren sind solche Objekte wie Holzsplitter, Eisenspäne und Kuhhornstücke in einen museumswürdigen Zustand eingetreten.

Der im Zuge der Digitalisierungsprozesse deutlich gewordene zeitliche Abstand bringt Objekte zu Bewusstsein, die zuvor nicht als museal galten und auch nicht zu diesem Zweck angeschafft oder gepflegt wurden. Die Ob- 
jekte universitärer Sammlungen unterliegen einer permanenten Bedeutungsumwandlung, die sie von Lehr- und Forschungsmaterialien zu historischen Sachzeugen macht. Heute stehen sie für die Geschichte der jeweiligen Bildungseinrichtung, sind Zeugnis einer (vergangenen) handwerklichen Meisterschaft oder geben Auskunft über die Geschichte der Wissenschaften. Faszinierend daran ist die Uneindeutigkeit des Prozesses: Das Besondere der universitären Sammlungen besteht zum einen darin, dass die Objekte gleichzeitige Bedeutungen besitzen können. Manche von ihnen sind nach wie vor in die Lehre eingebunden, während sie andernorts schon als museumswürdig angesehen werden. Dieses Changieren in der gegenwärtigen Situation macht sie aufregend und wertvoll: aufregend, weil man an ihnen die Entstehung des Historischen in seinen Facetten beobachten kann; wertvoll, weil sie Anlass geben zu zahlreichen Forschungsmöglichkeiten, die sich erst durch die brüchige Bedeutung der Dinge ergeben. Zum anderen schreiben sie sich erneut in den Forschungs- und Lehrzyklus einer Universität ein: diesmal als materielle Sachzeugen und als historische Quellen für eine wissenschaftshistorische Forschung, die über die lokalen Traditionen und eine Institutionengeschichte der Alma Mater weit hinausreichen kann. Hierin liegt das gemeinsame Potenzial der Sammlungen, nämlich zur Beantwortung der Frage nach den Herstellungsweisen von Wissenschaft beitragen zu können. Es ist deshalb keine bessere Schnittstelle für die Vermittlung und Sichtbarmachung einer sich rasant ändernden Wissenschaftskultur denkbar. Will man der aktuellsten und wichtigsten Verbindung von Wissensproduktion und Wissensdarstellung nachgehen, muss man hier beginnen.

\section{Danksagung}

Ich danke Anette Michels für Hinweise und Kommentare.

\section{Anmerkung}

1 Vgl. dazu den Artikel von Klaus Hentschel, „Bitte nicht wegwerfen“, Physik Journal, März 2007, 3; Für diesen Hinweis danke ich Roland Wittje.

Anke te Heesen

Ludwig-Uhland-Institut für Empirische Kulturwissenschaft

Universität Tübingen

Burgsteige 10

72070 Tübingen

E-Mail: anke.te-heesen@uni-tuebingen.de 